\title{
Using the finite domain remnant of the continuous spectrum to examine integrability: Effect of boundary conditions
}

\author{
Panayotis G. Kevrekidis ${ }^{1}$ and Niurka R. Quintero ${ }^{2}$ \\ ${ }^{1}$ Department of Mathematics and Statistics, University of Massachusetts, Amherst, Massachusetts 01003-4515, USA \\ ${ }^{2}$ Departamento de Física Aplicada I, Escuela Universitaria Politécnica, Universidad de Sevilla, Virgen de África 7, 41011 Sevilla, Spain \\ and Instituto Carlos I de Física Teórica y Computacional, Universidad de Granada, E-18071 Granada, Spain
}

(Received 11 December 2002; revised manuscript received 30 June 2003; published 23 September 2003)

\begin{abstract}
The aim of this work is to propose a method for testing the integrability of a model partial differential (PDE) and/or differential difference equation (DDE), by examining it in a finite but large domain. For monoparametric families of PDE/DDE's, that are known to possess isolated integrable points, we find that very special features occur in the finite domain remnant of the continuous ("phonon") spectrum at these "singular" points. We identify these features in the case example of a PDE and a DDE (that sustain front and pulselike solutions, respectively) for different types of boundary conditions. The key finding of the work is that such spectral features are generic near the singular, integrable points and hence we propose to explore a given PDE/DDE in a finite but large domain for such traits, as a means of assessing its potential integrability.
\end{abstract}

DOI: 10.1103/PhysRevE.68.036612

PACS number(s): 05.45.-a, 02.30.Ik

\section{INTRODUCTION}

Integrable models of partial differential (PDE) and differential difference (DDE) equations have been a topic of intense investigation over the past few decades [1-3]. The main reason for this, except for the wide variety of physical applications that can be described by integrable or nearintegrable systems, is that the special case of integrable models can be analyzed completely by means of the inverse scattering transform $[1,4]$. This can then serve as a starting point for perturbative treatment of near-integrable systems.

In the process of these developments, a number of techniques have been developed for assessing integrability in continuous [5] or discrete [6] settings (or applicable to both [7]). An interesting feature of these "tests" is that they are necessary (but not sufficient) conditions for integrability. Hence, if a model equation fails such a criterion, it is nonintegrable, but if it passes, it may or may not be integrable. In a sense, this suggests that we still do not understand the essential ingredients that render a system completely integrable. Of course, should a Lax pair be identified and the inverse scattering mechanism be applied, we know that the system is integrable, but it would certainly be desirable (as is clear from all the above effort to create "integrability tests") to have a mechanistic ("black box") type of criterion to assess that.

We, of course, do not claim to be providing a full answer to this question in the present work. However, we will try to give a number of useful hints that may lead to partial answers to the above questions and may provide some intuition in the effort to construct such mechanistic criteria.

Our tool of choice will be the use of different sets of boundary conditions (BC) to examine the spectrum of the linearization around the nonlinear coherent structure that the PDE/DDE of interest supports. Notice that the effect of boundary conditions in related contexts has been studied in a number of references; see, e.g., Ref. [8], and references therein. However, in all of these works the effects of the BC to the point spectrum were assessed and moreover, this was not done in direct connection with issues of integrability. Here we will, instead, focus on the continuous spectrum; in fact, since we will be dealing with finite but large domains, we will center our attention around the discrete spectrum remnant that "becomes" the continuous spectrum in the infinite domain limit. In the finite domain case, the (formerly continuous) spectrum becomes discrete due to the quantization of the wave numbers, imposed by the boundary conditions (see, e.g., Sec. II below). It is exactly this discrete remnant of the continuous spectrum, that we aim at examining here, to elucidate its interesting properties in integrable versus nonintegrable settings.

In the present work, we focus on two model problems, to establish our findings and demonstrate their generality. The models are selected as one-parameter families of equations such that one member of the family is an integrable system. Moreover, in illustrating the generality of the conclusions, they are selected in a form such that one model corresponds to a PDE, while the other to a DDE, so that one is kink bearing, while the other is pulse bearing. The models of interest will be the parametrically modified sine-Gordon equation [often also called the Peyrard-Remoissenet (PR) model] $[9,10]$ and a modified version of the discrete nonlinear Schrödinger (DNLS) model (occasionally called the Salerno model) [11]. The former PDE reads

$$
\phi_{t t}-\phi_{x x}=-\frac{d U}{d \phi}, \quad U(\phi, r)=\frac{(1-r)^{2}[1-\cos (\phi)]}{1+r^{2}+2 r \cos (\phi)}
$$

in the infinite domain $|x|<\infty$ and with $|r|<1$; while the latter DDE is of the form

$$
i \dot{u}_{n}=-\Delta_{2} u_{n}-\left|u_{n}\right|^{2}\left[2 \epsilon u_{n}+(1-\epsilon)\left(u_{n+1}+u_{n-1}\right)\right] .
$$

The most well known among these monoparametric families of models are the sine-Gordon equation [Eq. (1), for $r=0$ ] which is relevant to superconductivity and charge density 
waves among other applications [2] and the experimentally realizable discrete nonlinear Schrödinger equation [12] of $\epsilon$ $=1$, as well as its integrable, so-called Ablowitz-Ladik [13] counterpart for $\epsilon=0$ in the case of Eq. (2).

Notice that for the PDE, the subscripts denote partial derivatives of the field, while for the DDE, the overdot denotes temporal derivative, $\Delta_{2} u_{n} \equiv C\left(u_{n+1}-2 u_{n}+u_{n-1}\right)$, where $C=1 /(\Delta x)^{2}$ is a constant determined by the lattice spacing $\Delta x$; the subscript $n$ denotes the lattice site index. In the former case, there exist kinklike solutions which have been detailed in Refs. $[9,10]$, while in the latter, the field is complex and there exist pulselike solutions of the form $u_{n}$ $=\exp (i \Lambda t) v_{n}$, where $\Lambda$ is the frequency of the solutions and $v_{n}$ its (real) exponentially localized spatial profile $[11,12]$.

In the PDE, linearization around a state $\phi_{0}(x)$, using the ansatz $\phi=\phi_{0}(x)+\delta \exp (i \omega t) f(x)$ into Eq. (1), yields to $O(\delta)$ the linearization equation

$$
f_{x x}+\left[\omega^{2}-U^{\prime \prime}\left(\phi_{0}, r\right)\right] f=0 .
$$

Notice that when $r=0$ (in the infinite domain limit), $\phi_{0}(x)$ $=4 \arctan [\exp (x)]$ is the static kink solution of the sG equation and for this function, the Sturm-Liouville problem (3) can be exactly solved [14] yielding one discrete mode (Goldstone mode) at $\omega=0$ and the continuous spectrum represented by the phonons,

$$
\omega_{k}=\sqrt{1+k^{2}}, \quad f_{k}(x)=\frac{\exp (i k x)}{\sqrt{2 \pi} \omega_{k}}[k+i \tanh (x)],
$$

for all values of $k$. For $r \neq 0$, neither the static solution nor the linearization spectrum are explicitly available in the infinite domain limit.

Analogously to the PDE, for the linear stability analysis of DDE (2) we insert $\exp (i \Lambda t)\left[v_{n}+\delta\left(U_{n} e^{-i \omega t}+W_{n} e^{i \omega^{\star} t}\right)\right]$ into Eq. (2). We thus obtain to $O(\delta)$ the following eigenvalue problem for $\left\{\omega,\left\{U_{n}, W_{n}^{\star}\right\}\right\}$ :

$$
\begin{gathered}
\omega\left(\begin{array}{c}
U_{n} \\
W_{n}^{\star}
\end{array}\right)=\mathcal{L}\left(\begin{array}{c}
U_{n} \\
W_{n}^{\star}
\end{array}\right), \quad \mathcal{L}=\left(\begin{array}{cc}
A & B \\
-B & -A
\end{array}\right), \\
A_{m n}=\left[\Lambda+2 C-\left\{4 \epsilon v_{n}^{2}+(1-\epsilon) v_{n}\left[v_{n+1}+v_{n-1}\right]\right\}\right] \delta_{m, n} \\
+\left[(1-\epsilon) v_{n}^{2}-C\right]\left(\delta_{m, n+1}+\delta_{m, n-1}\right), \\
B_{m n}=-v_{n}\left[2 \epsilon v_{n}+(1-\epsilon)\left(v_{n+1}+v_{n-1}\right)\right] \delta_{m, n},
\end{gathered}
$$

where the stars denote complex conjugation.

The paper is organized as follow. In the following section we obtain an approximate solution for the Sturm-Liouville problem (3) by imposing different types of boundary conditions in the finite domain of length $L$. The obtained results are compared with the numerical computations in Sec. III, where we also computed the solution of Eq. (5). Finally, we summarize our findings and present our conclusions in Sec. IV.

\section{ANALYTICAL APPROXIMATION}

In this section we solve approximately Eq. (3) when $|x|$ $<L / 2$, where $L$ is the finite (but large enough) length of the system. Notice that our results will be generically true, if $L$ is chosen large enough. By large enough here, we mean a domain size which is many times (at least 10) larger than the characteristic length of the solitary wave (kink or pulse) that we will examine inside this domain. We will take into account different kinds of $\mathrm{BC}$, in particular, free

$$
f_{x}(-L / 2)=0, f_{x}(L / 2)=0
$$

fixed

$$
f(-L / 2)=0, \quad f(L / 2)=0,
$$

and antiperiodic boundary condition (aPBC)

$$
f_{x}(-L / 2)=-f_{x}(L / 2), \quad f(-L / 2)=-f(L / 2) .
$$

First we consider the integrable case, $r=0$, and we show that for the first phonon modes, the eigenfrequencies $\tilde{\omega}_{n}^{\text {free }}$ $=\tilde{\omega}_{n-1}^{\text {fixed }}$ and $\tilde{\omega}_{n}^{a p}$ have a double multiplicity (we will denote with tilde the analytical, approximated eigenfrequencies). To proceed, we use the exact solution of problem (3) for $r=0$ in the infinite domain. We would like to stress that if we change the infinite domain by a finite one, with a given $\mathrm{BC}$, we will still have an infinite number of eigenfrequencies, ${ }^{1}$ but for the allowed wave numbers $k$ [15]. In order to calculate approximately these allowed wave numbers, we proceed as in Ref. [16]. Notice that $f_{k}(x)=F_{k}(x)+i G_{k}(x)$, where

$$
\begin{aligned}
& F_{k}(x)=\frac{k \cos (k x)-\sin (k x) \tanh (x)}{\sqrt{2 \pi} \omega_{k}}, \\
& G_{k}(x)=\frac{\cos (k x) \tanh (x)+k \sin (k x)}{\sqrt{2 \pi} \omega_{k}} .
\end{aligned}
$$

Then the solution of Eq. (3), with $r=0$, related to the phonon contribution is represented by the linear superposition of all the odd $\left[G_{k}(x)\right]$ and even $\left[F_{k}(x)\right]$ phonon modes

$$
\psi(x, t)=\sum_{k}\left[a_{k}(t) F_{k}(x)+b_{k}(t) G_{k}(x)\right] .
$$

Imposing free BC for each phonon mode of Eq. (11) we obtain that the first wave numbers satisfy

$$
\begin{aligned}
& a_{k}(t)\left[\sin (k L / 2)\left[k^{2}+\cosh ^{-2}(L / 2)\right]+k \cos (k L / 2) \tanh (L / 2)\right] \\
& \quad=0, \\
& b_{k}(t)\left[\cos (k L / 2)\left[k^{2}+\cosh ^{-2}(L / 2)\right]-k \sin (k L / 2) \tanh (L / 2)\right] \\
& \quad=0 .
\end{aligned}
$$

\footnotetext{
${ }^{1}$ Note that this is true for the continuum problem of Eq. (3), but would no longer be true for the discrete one of Eq. (5).
} 
The solutions of these transcendental equations yield the allowed values of $k$. We can solve these approximately if we consider $L \gg 1$. Then, we find that

$$
k_{n, 0}^{\text {free }}=\frac{n-1}{L} \pi, \quad n=1,2,3, \ldots, \quad n \ll L,
$$

where the zero subscript denotes that we are dealing with the unperturbed case $r=0$, and its corresponding eigenfunctions are related with the odd functions $G_{n}(x)$ for the odd numbers $n$ and with the even functions $F_{n}(x)$ for the even numbers $n$. Hence, the first eigenfrequencies are represented by

$$
\tilde{\omega}_{n, 0}^{\text {free }}=\sqrt{1+\left(\frac{n-1}{L} \pi\right)^{2}}, \quad n=1,2, \ldots, \quad n \ll L .
$$

Analogously, for fixed $\mathrm{BC}$ the following relations hold;

$$
\begin{gathered}
a_{k}(t)[k \cos (k L / 2)-\sin (k L / 2) \tanh (L / 2)]=0, \\
b_{k}(t)[\cos (k L / 2) \tanh (L / 2)+k \sin (k L / 2)]=0 .
\end{gathered}
$$

Then, for large enough $L$, we find that

$$
k_{n, 0}^{\text {fixed }}=\frac{n}{L} \pi, \quad n=1,2,3, \ldots, \quad n \ll L,
$$

and so,

$$
\tilde{\omega}_{n, 0}^{\text {fixed }}=\sqrt{1+\left(\frac{n}{L} \pi\right)^{2}}, \quad n=1,2, \ldots, \quad n \ll L,
$$

where the odd (even) numbers $n$ are related with the odd $G_{n}(x)$ [even $F_{n}(x)$ ] eigenfunctions.

Remark 1. By comparing expressions (15) and (19) we observe that in the integrable case $(r=0) \tilde{\omega}_{n}^{\text {free }}=\tilde{\omega}_{n-1}^{\text {fixed }}$ for the first few eigenfrequencies.

Now by imposing aPBC in each phonon mode of Eq. (11) and taking into account the symmetry properties of $F_{k}(x)$, $G_{k}(x)$ and their derivatives, the equations that the wave number satisfies can be reduced to

$$
\begin{gathered}
a_{k} F_{k}(L / 2)=0, \\
b_{k} \frac{\partial G_{k}}{\partial x}(L / 2)=0 .
\end{gathered}
$$

Notice that Eqs. (20) and (21) coincide with Eqs. (16) (i.e., the first equation for fixed $\mathrm{BC}$ ) and (13) (i.e., the second equation for free BC), respectively. The solutions of Eqs. (20) and (21) are given by

$$
\begin{gathered}
k_{n, 0}^{a p}=\frac{2(n-1) \pi}{L}, \quad n=2,3, \ldots, \quad n \ll L, \\
k_{n, 0}^{a p}=\frac{2(n-1) \pi}{L}, \quad n=1,2,3, \ldots, \quad n \ll L,
\end{gathered}
$$

respectively, and their eigenfunctions correspond to the even $F_{n}(x)$ and odd $G_{n}(x)$. Then, the first eigenfrequencies are represented by

$$
\begin{gathered}
\tilde{\omega}_{n, 0}^{a p}=\sqrt{1+\left(\frac{2(n-1) \pi}{L}\right)^{2}}, \quad n=2,3 \ldots, n \ll L, \\
\tilde{\omega}_{n, 0}^{a p}=\sqrt{1+\left(\frac{2(n-1) \pi}{L}\right)^{2}}, \quad n=1,2, \ldots, \quad n \ll L .
\end{gathered}
$$

This means that the even (odd) modes for aPBC $\left\{\tilde{\omega}_{n}^{a p}, a_{n}(t) F_{n}(x)\right\}\left[\left\{\tilde{\omega}_{n}^{a p}, b_{n}(t) G_{n}(x)\right\}\right]$ coincide with the even modes for fixed BC (odd modes for free BC).

Remark 2. From relations (24) and (25) we conclude that for the integrable case and $\mathrm{aPBC}$ the eigenfrequencies have multiplicity 2.

The analysis of the Sturm-Liouville problem (3) for the nonintegrable case, $r \neq 0$, becomes more complicated since $\phi_{0}(x)$ is the exact kink solution of Eq. (1) and this function is only known in the implicit form [9] (even for the infinite domain problem). So, instead of solving this equation we calculate approximately the solution of

$$
\left[\frac{d^{2}}{d x^{2}}-V(x)-r W(x)+E\right] f=0,
$$

where $\quad V(x)=-2 / \cosh ^{2}(x), \quad W(x)=8 \tanh (x)[x$ $-5 \tanh (x)] / \cosh ^{2}(x)$, and $E=\omega^{2}-\omega_{p h}^{2}$ with $\omega_{p h}=(1$ $-r) /(1+r)[10,17]$. This eigenvalue problem is obtained in two steps: first we find a solution for small $r$ of Eq. (1), through the perturbative expansion $\phi(x, t)=\phi_{s G}(x)$ $+r \phi_{1}(x)+O\left(r^{2}\right)$, where $\phi_{s G}(x)$ is the static sG kink and second we linearize Eq. (1) around the obtained solution up to order of $r$, so we insert $\phi(x, t)=\phi_{s G}(x)+r \phi_{1}(x)$ $+\delta\left[f(x) \exp (i \omega t)+f^{\star}(x) \exp (-i \omega t)\right]$ into Eq. (1) and consider the equation that arises to $O(\delta)$ and obtain Eq. (26). Arguably, this approach fails to capture the corrections to the tail of the wave due to domain finiteness. However, as argued in Ref. [8], the latter are exponentially small in the length of the domain. Hence, as will also be justified a posteriori, here we capture the leading order dependence in $L$, as well as the leading order effect of $r$ [see, e.g., Eqs. (31)-(33) below]. Then, following the procedure of the perturbation methods for linear eigenvalue problem suggested in Ref. [18], we assume the solution of Eq. (26) as

$$
\begin{gathered}
E_{n}=E_{n, 0}+r E_{n, 1}+O\left(r^{2}\right), \\
f_{n}(x)=f_{n, 0}(x)+r f_{n, 1}(x)+O\left(r^{2}\right),
\end{gathered}
$$

where the first subscript in the functions, $n$, denotes the order of the phonon modes (for $r<0$ this subscript can also denote the internal mode), the second one corresponds to the order of perturbation. By inserting these expansions in Eq. (26) and equating and collecting the terms of the same order in $r$, we obtain for $O\left(r^{0}\right)$, 
TABLE I. For positive and small value of $r=0.02$, we compare the first eigenfrequencies, obtained perturbatively, $\tilde{\omega}_{n}$, with the ones computed by solving the original Eqs. (1) and (3), $\omega_{n}$.

\begin{tabular}{ccccccc}
\hline \hline$n$ & $\omega_{n}^{\text {free }}$ & $\tilde{\omega}_{n}^{\text {free }}$ & $\omega_{n}^{\text {fixed }}$ & $\tilde{\omega}_{n}^{\text {fixed }}$ & $\omega_{n}^{a p}$ & $\tilde{\omega}_{n}^{a p}$ \\
\hline 1 & 0.96117 & 0.96192 & 0.96234 & 0.96352 & 0.96117 & 0.96192 \\
2 & 0.96132 & 0.96129 & 0.96293 & 0.96284 & 0.96293 & 0.96284 \\
3 & 0.96436 & 0.96514 & 0.96729 & 0.96760 & 0.96436 & 0.96514 \\
4 & 0.96560 & 0.96541 & 0.96933 & 0.96899 & 0.96933 & 0.96899 \\
5 & 0.97118 & 0.97126 & 0.97604 & 0.97573 & 0.97118 & 0.97126 \\
6 & 0.97411 & 0.97358 & 0.97989 & 0.97915 & 0.97991 & 0.97915 \\
\hline \hline
\end{tabular}

$$
\left[\frac{d^{2}}{d x^{2}}-V(x)+E_{n, 0}\right] f_{n, 0}=0,
$$

and for the next order correction $O\left(r^{1}\right)$,

$$
\left[\frac{d^{2}}{d x^{2}}-V(x)+E_{n, 0}\right] f_{n, 1}=\left[W(x)-E_{n, 1}\right] f_{n, 0} .
$$

Notice that Eq. (29) corresponds to the integrable case $r$ $=0$ already solved for free [Eq. (15)], fixed [Eq. (19)], and antiperiodic BC [see Eqs. (24) and (25)]. Notice also that $E_{n, 0}=\widetilde{\omega}_{n, 0}^{2}-1=k_{n, 0}^{2}$ and that its corresponding eigenfunction $f_{n, 0}(x)$ is related either with the odd $G_{n}(x)$ or even $F_{n}(x)$. Then, for different boundary conditions, the eigenfrequencies of Eq. (26) are determined by

$$
\begin{gathered}
\tilde{\omega}_{n}^{\text {free }}=\sqrt{\omega_{p h}^{2}+\left(k_{n, 0}^{\text {free }}\right)^{2}+r E_{n, 1}^{\text {free }},} \\
\tilde{\omega}_{n}^{\text {fixed }}=\sqrt{\omega_{p h}^{2}+\left(k_{n, 0}^{\text {fixed }}\right)^{2}+r E_{n, 1}^{\text {fixed }},} \\
\tilde{\omega}_{n}^{a p}=\sqrt{\omega_{p h}^{2}+\left(k_{n, 0}^{a p}\right)^{2}+r E_{n, 1}^{a p},}
\end{gathered}
$$

where $k_{n, 0}^{\text {free }}, k_{n, 0}^{\text {fixed }}$, and $k_{n, 0}^{a p}$ are given by Eqs. (14), (18), and (22) and (23), respectively.

The solution of the eigenvalue $E_{n, 1}$ for the first-order correction is given by

$$
E_{n, 1}=\frac{\int_{-L / 2}^{L / 2} d x f_{n, 0}(x) W(x) f_{n, 0}(x)}{\int_{-L / 2}^{L / 2} d x f_{n, 0}^{2}(x)}
$$

The integrals involved in Eq. (34) can be computed numerically for different BC and different values of $r(|r| \ll 1)$, then we can calculate the approximated eigenfrequencies in each case. We can now compare these results with the numerical solutions of Eq. (3) [for details on the numerical methods/ results, we refer the reader to Sec. III].

From the data of the Tables I and II we observe an oscillatory behavior of $\tilde{\omega}_{n}^{\text {free }}-\tilde{\omega}_{n-1}^{\text {fixed }}$ for the first phonon's modes for $r \neq 0$. We also notice that the eigenvalues for aPBC lose their double multiplicity that existed in the case of the integrable equation.

It is also worth noting that these features are typically observable in the third decimal digit of the corresponding eigenfrequencies. On the other hand, the difference (well justified within the approximations mentioned above) between the theoretical and numerical predictions for the individual eigenfrequencies is typically in the fourth or fifth decimal digit. Hence, the observations of the previous paragraph are systematic and in agreement with the theoretical predictions.

\section{NUMERICAL RESULTS AND DISCUSSIONS}

To find the numerical solution of Eqs. (1) and (3), we discretize the equations in a numerical mesh for a finite domain. The mesh consists of the $N+1$ points $x_{j}=\{-L / 2$ $+j \Delta x, \quad j=0,1,2, \ldots, N\}$ defined in the finite length $L$ of the system $(\Delta x=L / N)$. Notice that since, in this case, we wish to emulate the behavior of the PDE, $\Delta x$ is very fine (typically 0.05$)$, and the robustness of the findings upon variation of the (small) $\Delta x$ has been verified. When we compute the solution either of the PDE or of the linearization equation, we consider three different types of BC (6)-(8). We would like to remark that this kind of discretization of the SturmLiouville problem (3) only affects the last phonon modes, so we can compare the behavior of the first phonon modes obtained in the preceding section with the numerical solution of Eq. (3). In both cases, the distributions of these eigenfrequencies are determined by the parameter $r$ and by the different boundary conditions in the finite domain.

TABLE II. We provide the same comparison as in the previous table for a negative value of $r=$ -0.02 . Here, $\widetilde{\Omega}_{i}$ and $\Omega_{i}$ represent the internal mode calculated by the perturbation method and computed by numerical solution of Eq. (3), respectively.

\begin{tabular}{ccccccc}
\hline \hline$n$ & $\omega_{n}^{\text {free }}$ & $\tilde{\omega}_{n}^{\text {free }}$ & $\omega_{n}^{\text {fixed }}$ & $\tilde{\omega}_{n}^{\text {fixed }}$ & $\omega_{n}^{a p}$ & $\widetilde{\omega}_{n}^{a p}$ \\
\hline & $\Omega_{i}=1.03560$ & $\widetilde{\Omega}_{i}=1.03977$ & $\Omega_{i}=1.03560$ & $\widetilde{\Omega}_{i}=1.03924$ & $\Omega_{i}=1.03560$ & $\widetilde{\Omega}_{i}=1.03977$ \\
1 & 1.04131 & 1.04129 & 1.04278 & 1.04271 & 1.04156 & 1.04058 \\
2 & 1.04156 & 1.04058 & 1.04367 & 1.04303 & 1.04278 & 1.04271 \\
3 & 1.04524 & 1.04506 & 1.04866 & 1.04835 & 1.04694 & 1.04625 \\
4 & 1.04694 & 1.04625 & 1.05125 & 1.05057 & 1.04867 & 1.04835 \\
5 & 1.05306 & 1.05257 & 1.05838 & 1.05770 & 1.05657 & 1.05564 \\
\hline \hline
\end{tabular}




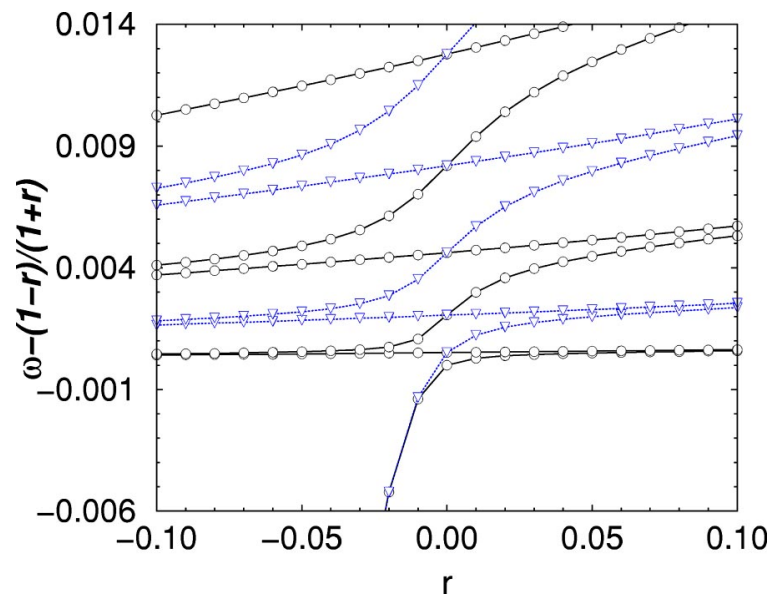

FIG. 1. Comparison of the eigenfrequencies of the (discrete remnant of the) continuous spectrum for fixed and free $\mathrm{BC}$ : We have plotted the difference between the eigenfrequencies computed from Eq. (3) and $\omega_{p h}=(1-r) /(1+r)$ vs $r$. The circles joined by solid line (free BC) represent how far the frequencies are from the lower phonon mode. The triangles joined by dotted lines correspond to fixed BC.

We also compute the solutions of DDE (2) and Eq. (5) using 200 points and $\Delta x=0.75$. The $\mathrm{BC}$ are defined analogously through $U_{0}=U_{1}, U_{N}=U_{N-1}, W_{0}=W_{1}$, and $W_{N}$ $=W_{N-1}$ for free BC. For fixed BC: $U_{0}=0, U_{N}=0, W_{0}$ $=0$, and $W_{N}=0$, while for periodic BC: $U_{0}=U_{N-1}, U_{N}$ $=U_{1}, W_{0}=W_{N-1}$, and $W_{N}=W_{1}$.

Our results when the parameter of the PR potential or $\epsilon$ in the DDE are varied can be summarized in Figs. 1-6.

From the above results, the following conclusions can be drawn.

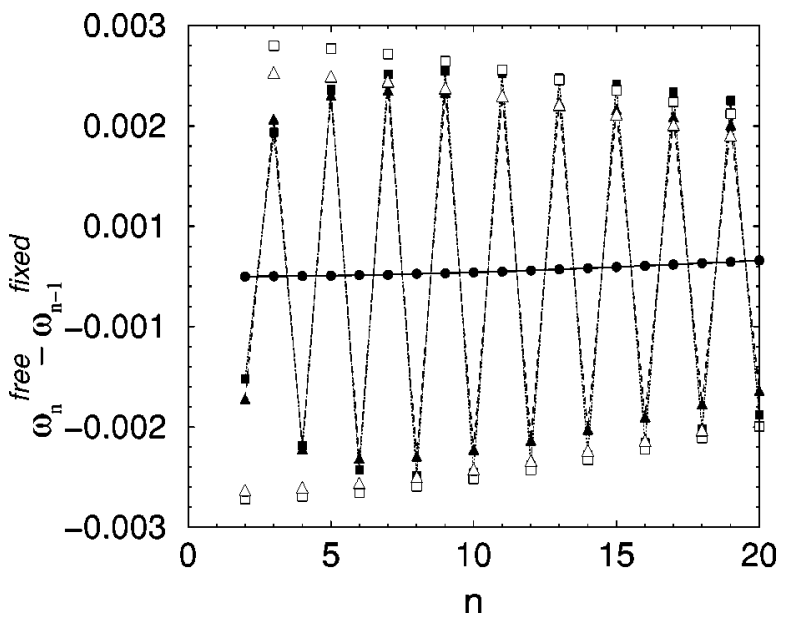

FIG. 2. The difference between the first frequencies for free and fixed BC, $\omega_{n}^{\text {free }}-\omega_{n-1}^{\text {fixed }}(2 \leqslant n \leqslant 20)$, is plotted as a function of the wave number for $r=0$ (circles joined by solid line), $r=-0.02$ (triangles joined by dashed line), and $r=0.02$ (squares joined by dotted line). The open triangles $(r=-0.02)$ and squares $(r=0.02)$ represent the differences between the frequencies obtained by the perturbation theory, $\tilde{\omega}_{n}^{\text {free }}-\tilde{\omega}_{n-1}^{\text {fixed }}$, in the preceding section.

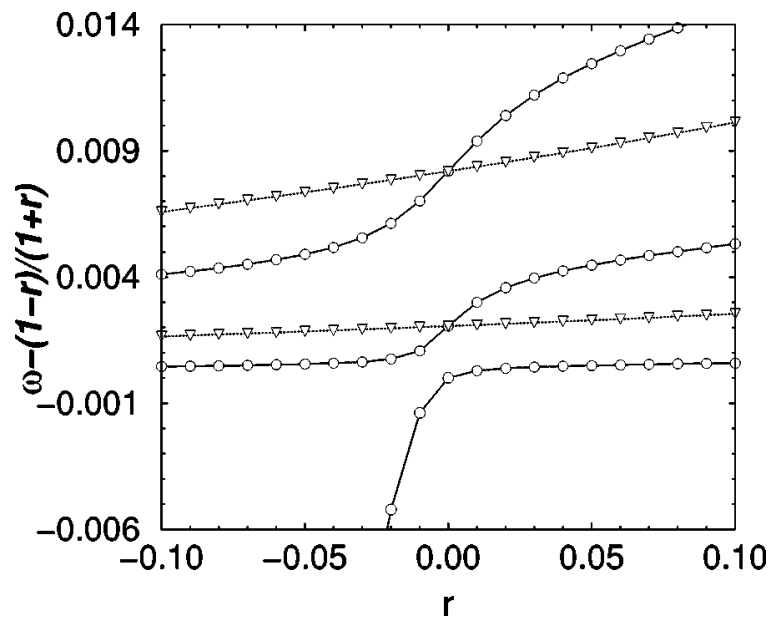

FIG. 3. Antiperiodic BC: the difference between the numerical eigenfrequencies computed from Eq. (3) and the band edge of the (formerly continuous) spectrum $\omega_{p h}=(1-r) /(1+r)$ is shown. Adjacent eigenmodes are given by circles joined by solid line and triangles joined by dotted line. The relevant internal mode is shown by circles joined by solid line (the first curve from below).

(1) For fixed BC, the band edge frequency is prohibited. Hence, we compare $\omega_{n}^{\text {free }}$ with $\omega_{n-1}^{\text {fixed }}$. We find that for small wave numbers, fixed and free $\mathrm{BC}$ eigenfrequencies practically coincide only in the integrable case, whereas for the nonintegrable case we observe an oscillatory behavior of this function [see Figs. 1 and 2]. In Fig. 2 we also show the oscillatory behavior of $\tilde{\omega}_{n}^{\text {free }}-\tilde{\omega}_{n-1}^{\text {fixed }}$, obtained from the perturbation theory, for $r=-0.02$ (open triangles) and $r=0.02$ (open squares).

(2) For antiperiodic BC, the spectrum comprises of modes coming alternately from the free and fixed BC. This seems natural as the free boundary conditions select eigenmodes

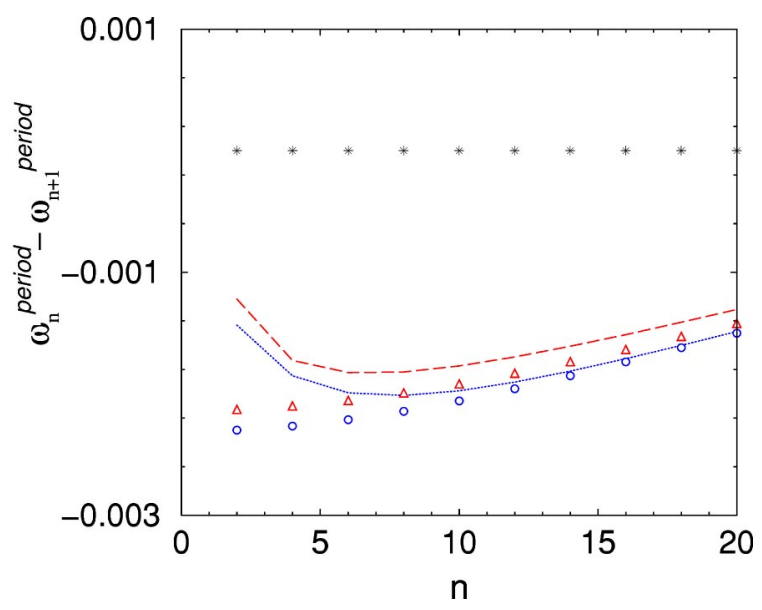

FIG. 4. Antiperiodic BC: We show the difference between $\omega_{n}^{\text {period }}-\omega_{n+1}^{\text {period }}$ vs $n(n=2,4, \ldots, 20)$. The stars practically at zero for all $n$ represent the integrable system $(r=0)$, whereas the long-dashed $(r=-0.02)$ and dotted $(r=0.02)$ lines correspond to nonintegrable cases (numerical results). With triangles $(r=$ $-0.02)$ and circles $(r=0.02)$ we plot the eigenfrequencies obtained from the perturbation theory (analytical results). 


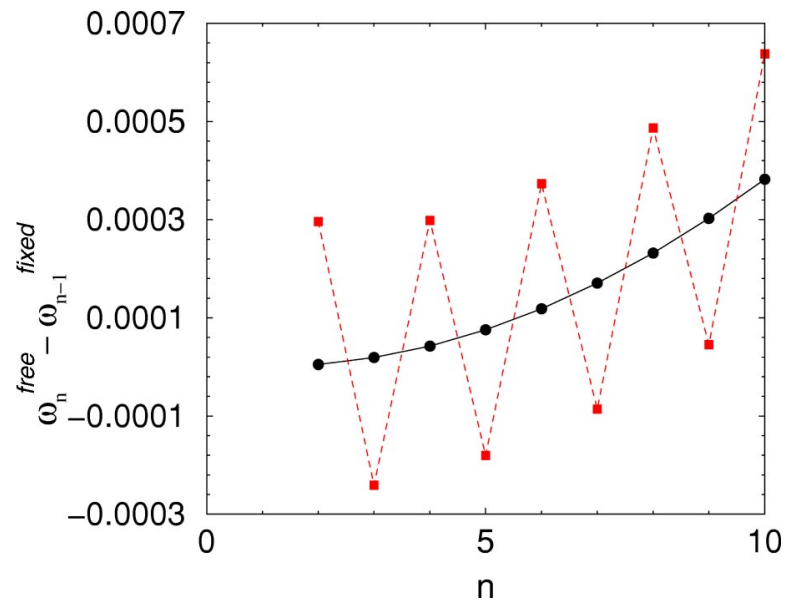

FIG. 5. The oscillatory behavior of $\omega_{n}^{\text {free }}-\omega_{n-1}^{\text {fixed }}$ in the nonintegrable case ( $\epsilon=0.1$ ) is shown for the first wave numbers (see the squares joined by dashed line). The circles joined by solid line are the results for the integrable system $(\epsilon=0)$.

symmetric at the boundary, the fixed ones select modes antisymmetric at the boundary, while the antiperiodic BC allow for both (cf. Figs. 3 and 4).

(3) An additional feature, equally important as (1) (especially in view of its potential predictive power) is the fact that for the integrable case of $r=0$, antiperiodic BC essentially imply the presence of double eigenvalues. The difference between the two eigenvalues is $O\left(10^{-9}\right)$ for all pairs (except for the cutoff, discretization induced phenomena at the upper end of the spectrum which are irrelevant). This is in sharp contrast (in particular, for small wave numbers), to even mild breakings of integrability, as can be inferred from Fig. 4.

(4) Statements (1) and (3) above can be used in predictive form and constitute the criterion (algorithm) set forth in this work: for a given PDE/DDE model, we find the steady state coherent structure (i.e., solitary wave) in a finite but large domain. This can be done, e.g., by finding the exact solution of an ODE or numerically performing a Newton-type algorithm. Linearize around the exact, finite domain solution and study, in particular, the small wave numbers, close to the lower edge of the spectrum (we assume that the problem is monoparametric in what follows, but it is clear that the application of the criterion does not require that). If for a critical/singular value of the parameter the fixed $\mathrm{BC}$ and free $\mathrm{BC}$ (small $k$ ) eigenvalue spectra (of the remnant of what for the infinite domain was the continuous spectrum) essentially coincide and the multiplicity of antiperiodic BC eigenvalues becomes double, then the model for this unique value of the parameter can be "strongly suspected" to be integrable. We use the above expression, as we provide no rigorous proof, but only supporting (but rather universal in distinct models with distinct features/solutions) numerical evidence for this statement.

(5) We have also tested the validity of these results in Eq. (2), in the vicinity of the integrable limit $\epsilon=0$, with similar conclusions [see Figs. 5 and 6]. Indeed, in Fig. 5 we observe the oscillatory behavior of $\omega_{n}^{\text {free }}-\omega_{n-1}^{\text {fixed }}$ in the nonintegrable

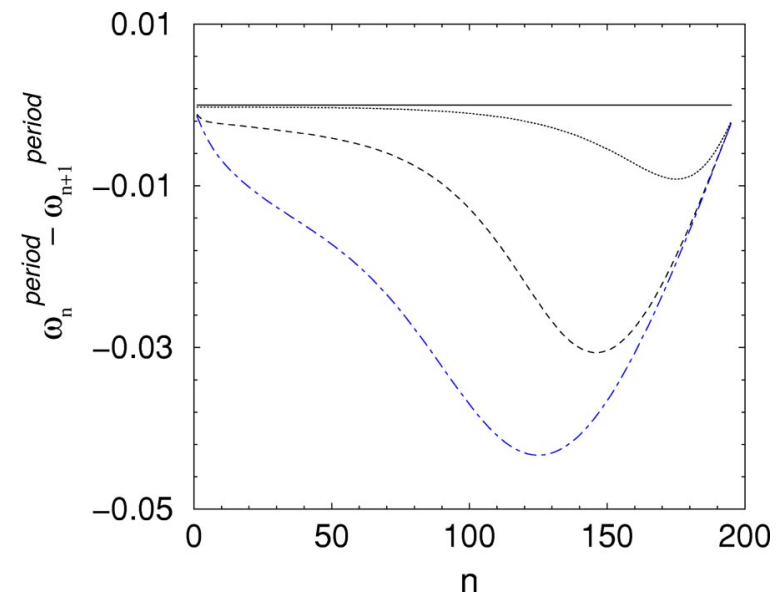

FIG. 6. Periodic BC for AL-DNLS (Ablowitz-Ladik DNLS) of Eq. (2): The solid line at zero represents the difference between two consecutive frequencies $(n=2,4, \ldots)$ for the integrable AL lattice $(\epsilon=0)$. The double multiplicity of the frequencies is destroyed as $\epsilon$ is increased (dotted, dashed, and dot-dashed lines represent the nonintegrable cases of $\epsilon=0.1,0.5,1$, respectively).

case, in Fig. 6, we show the case of periodic BC, where it can be clearly seen that it is only for the integrable case that the double eigenvalue multiplicity is obtained.

\section{CONCLUSIONS}

In conclusion, we have proposed and used a test for revealing the potential integrable nature of a given model problem. By varying the boundary conditions of a finite domain computation and examining the effects of such variations in the (continuous-turned-discrete) spectrum, we have revealed that the small wave numbers have singular ways of responding to the unique parameter values for which the model is integrable. These singular features [such as an approximate identification of fixed with free $\mathrm{BC}$ for small $k$ eigenvalues and the double multiplicity of eigenvalues for periodic (or antiperiodic) BC] can be used to identify and single out the integrable behavior. We have provided two model examples, respectively, for kinks and pulses and for a PDE and a DDE. Independently of the detailed structure of the model these properties have been identified as universal and have been supported also by analytical considerations. It would naturally be of interest to explore the potential usefulness of such a criterion in various more complex settings.

\section{ACKNOWLEDGMENTS}

We would like to thank Jesús Sánchez-Dehesa for the useful discussion on the perturbation theory in the SturmLiouville problem. This work has been supported by the Ministerio de Ciencia y Tecnología of Spain through Grant No. BFM2001-3878-C02 and by the Junta de Andalucía under Project No. FQM-0207 (N.R.Q.). It has also been partially supported by NSF under Grant No. DMS-0204585, a University of Massachusetts Faculty Research Grant and the Eppley Foundation for Research (P.G.K.). 
[1] M.J. Ablowitz and H. Segur, Solitons and the Inverse Scattering Transform (SIAM, Philadelphia, 1981).

[2] R.K. Dodd, J.C. Eilbeck, J.D. Gibbon, and H.C. Morris, Solitons and Nonlinear Wave Equations (Academic Press, London, 1982).

[3] A.C. Scott, Nonlinear Science (Oxford University Press, Oxford, 1999).

[4] L.D. Faddeev and L.A. Takhtajan, Hamiltonian Methods in the Theory of Solitons (Springer-Verlag, Berlin, 1987).

[5] P. Painlevé, C. R. Acad. Sci. (Paris) 130, 1112 (1900); A. Ramani, B. Grammaticos, and A. Bountis, Phys. Rep. 180, 159 (1989); R. Conte, e-print solv-int/9710020.

[6] B. Grammaticos, A. Ramani, and V. Papageorgiou, Phys. Rev. Lett. 67, 1825 (1991); A. Ramani, B. Grammaticos, and J. Hietarinta, ibid. 67, 1829 (1991); M. Bellon and C.-M. Viallet, e-print chao-dyn/9805006; J. Hietarinta and C. Viallet, Phys. Rev. Lett. 81, 325 (1998).

[7] P.G. Kevrekidis, Phys. Lett. A 285, 383 (2001).

[8] R.M. DeLeonardis, S.E. Trullinger, and R.F. Wallis, J. Appl. Phys. 51, 1211 (1980); P.G. Kevrekidis, I.G. Kevrekidis, and B.A. Malomed, ibid. 35, 267 (2002); K.Ø. Rasmussen, D. Cai,
A.R. Bishop, and N. Grønbech-Jensen, Phys. Rev. E 55, 6151 (1997).

[9] M. Remoissenet and M. Peyrard, J. Phys. C 14, L481 (1981); M. Peyrard and M. Remoissenet, Phys. Rev. B 26, 2886 (1982).

[10] N.R. Quintero and P.G. Kevrekidis, Physica D 170, 31 (2002).

[11] D. Cai, A.R. Bishop, and N. Gronbech-Jensen, Phys. Rev. Lett. 72, 591 (1994).

[12] See, e.g., P.G. Kevrekidis, K.Ø. Rasmussen, and A.R. Bishop, Int. J. Mod. Phys. B 15, 2833 (2001), and references therein.

[13] M.J. Ablowitz and J.F. Ladik, J. Math. Phys. 16, 598 (1975); 17, 1011 (1976).

[14] J. Rubinstein, J. Math. Phys. 11, 258 (1970).

[15] E.A. Coddington and N. Levinson, Theory of Ordinary Differential Equations (McGraw-Hill, New York, 1955).

[16] N.R. Quintero and P.G. Kevrekidis, Phys. Rev. E 64, 056608 (2001).

[17] Yu.S. Kivshar, D. Pelinovsky, T. Cretegny, and M. Peyrard, Phys. Rev. Lett. 80, 5032 (1998).

[18] C.M. Bender and S.A. Orszag, Advanced Mathematical Methods for Scientists and Engineers (McGraw-Hill, New York, 1978). 